\title{
A Questionnaire-Based Survey to Assess the Knowledge and Awareness of Denture Staining in Complete Denture Wearers
}

\author{
Pervez Cooper ${ }^{1} \quad$ Rakshith Hegde $^{1} \quad$ Chethan Hegde $^{1}$ \\ ${ }^{1}$ Department of Prosthodontics and Crown and Bridge, A.B. Shetty \\ Memorial Institute of Dental Sciences, Mangaluru, Karnataka, India
}

Address for correspondence Pervez Cooper, MDS (Student), Department of Prosthodontics and Crown and Bridge, A.B. Shetty Memorial Institute of Dental Science, Medical Sciences Complex, Deralakatte, Mangaluru 575018, Karnataka, India (e-mail: pervezcooper@hotmail.co.in).

\begin{abstract}
Background Knowledge of denture hygiene and staining is of importance for the edentulous patient to maintain a clean and hygienic prosthesis. However, many patients lack this basic knowledge. This survey aims at assessing the level of awareness among patients for the same.

Objective The aim and objective of this article are (1) to assess the knowledge of denture wearers on denture hygiene and maintenance, and (2) to gauge the awareness of denture wearers on denture staining.

Sample Selection Study sample will be consisting of 100 completely edentulous patients reporting to the Department of Prosthodontics in A. B. Shetty Memorial Institute of Dental Sciences.

Keywords

- denture hygiene

Results Majority of the patients lacked proper education with regards to complete denture staining and hygiene maintenance and also lacked the proper motivation to carry out denture hygiene maintenance practices on a daily basis.
\end{abstract}

\section{Introduction}

Oral hygiene is an important practice that needs to be maintained in our day to day lives. Daily effective oral hygiene measures should be practiced and pursued to maintain a good periodontal health. Failing to do that will indefinitely lead to the consequences of plaque accumulation. Dental plaque accumulates on the hard tissues as well as soft tissues of the oral cavity. Dental prosthesis including removable dentures is also unguarded against the peril of plaque accumulation; hence, oral hygiene maintenance is a life-long exercise.

Complete dentures constitute one of the most important treatment options in prosthodontics. Newly constructed dentures could be a disappointment to patients if they are ineffectual in maintaining proper denture hygiene. Complete dentures aid in the masticatory process, phonetics, and also restore an edentulous individual's smile. Besides these

received

DOI https://doi.org/

October 12, 2018

$10.1055 / \mathrm{s}-0039-1694803$ reasons, denture hygiene should be maintained for its longevity and aesthetic maintenance. It is important to take care of the dentures in a similar way we maintain the natural dentition, and sometimes, a little extra care may be needed to avoid staining on dentures.

Awareness is said to be the first step toward any change. A complete denture wearer's awareness of oral hygiene and denture hygiene maintenance is directly related to their lack of knowledge of the same or their ignorance. The purpose of this survey was to assess complete denture wearers' knowledge on denture hygiene maintenance and staining, and their awareness for the same. ${ }^{1-4}$

\section{Materials and Methods}

Sample consisted of 100 completely edentulous patients that reported to the Department of Prosthodontics of A. B. Shetty Memorial Institute of Dental Sciences. Patients already 
wearing complete dentures and who are physically and intellectually capable of maintaining denture cleanliness and hygiene were selected for the study. A self-explanatory questionnaire was given to the patients to collect the data which included questions relating to denture hygiene awareness and maintenance. Prior to data collection informed consent was taken from all the subjects. The results were analyzed based on the collective response of each question among the 100 patients.

The following questionnaire was distributed to the patients:

1. How long have you been wearing a denture?
a. Less than 6 months
b. 6 months -1 year
c. 1-5 years
d. More than 5 years

2. Do you drink coffee or tea regularly?
a. Yes
b. No

3. If yes, at what intervals do you drink?
a. 2 times a day
b. 3-5 times a day
c. More than 5 times a day

4. Do you consume aerated drinks?
a. Yes
b. No

5. If yes, what is the frequency of consumption of said drinks?
a. Daily
b. Frequently (more than once a week)
c. Occasionally

6. Do you have any of the following habits?
a. Alcohol consumption
b. Cigarette smoking
c. Tobacco chewing
d. None of the above

7. If yes, what is the frequency of those habits?
a. Daily
b. Frequently (more than once a week)
c. Occasionally

8. Do you clean your dentures?
a. Yes
b. No

9. At what intervals do you clean your dentures?
a. Once daily
b. Twice daily
c. Every alternate day

10. What method do you use to clean your dentures?
a. Denture cleaning solutions
b. Rinsing with water
c. Using toothpaste
d. Using detergents

11. Are you aware of any denture cleaning techniques?
a. Yes
b. No

12. If yes, what techniques have you been advised?
a. Soaking in salt water overnight
b. Using denture cleaning solutions
c. Professional cleaning by a dental technician

13. Do you suffer any of the following?
a. Oral infections
b. Oral mucosal ulcers
c. Halitosis (bad breath)

14. Are you aware that stained dentures could be a cause for the above discomforts?
a. Yes
b. No

15. Are you satisfied with your denture maintenance?
a. Yes
b. No

\section{Results}

About $32 \%$ of the patients were denture wearers for 6 months to 1 year. Approximately $20 \%$ of the patients had used dentures for less than 6 months. About 31\% of the patients had been wearing dentures for 1 to 5 years and approximately $17 \%$ of the patients were complete denture wearers for more than 5 years ( - Fig. 1). About $93 \%$ of patients consume coffee or tea regularly ( $\boldsymbol{- \text { Fig. }} \mathbf{2}$ ). Of the patients consuming tea or coffee, approximately $4 \%$ of the patients consumed tea or coffee more than 5 times a day. About $28 \%$ of patients consumed tea or coffee 3 to 5 times a day. Around $68 \%$ of the patients consumed tea or coffee only twice a day ( - Fig. $\mathbf{3}$ ). About $44 \%$ of the patients consumed aerated beverages

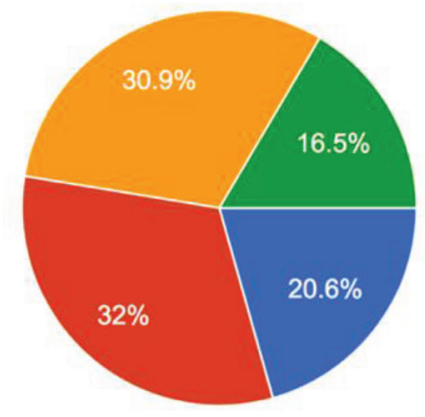

Fig. 1 Duration of denture usage. 
( - Fig. 4). Of the patients consuming aerated beverages, approximately $8 \%$ of patients consumed aerated beverages daily. About $20 \%$ of the patients consumed aerated beverages more than once a week and around $72 \%$ of the patients consumed aerated beverages occasionally ( - Fig. 5). Forty-seven

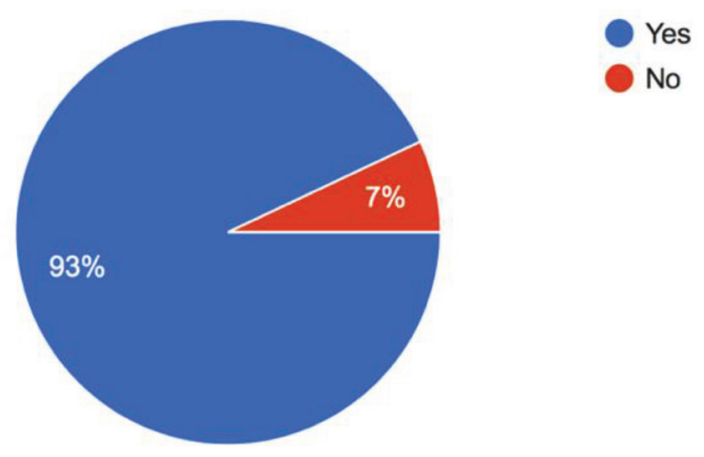

Fig. 2 Consumption of tea or coffee.

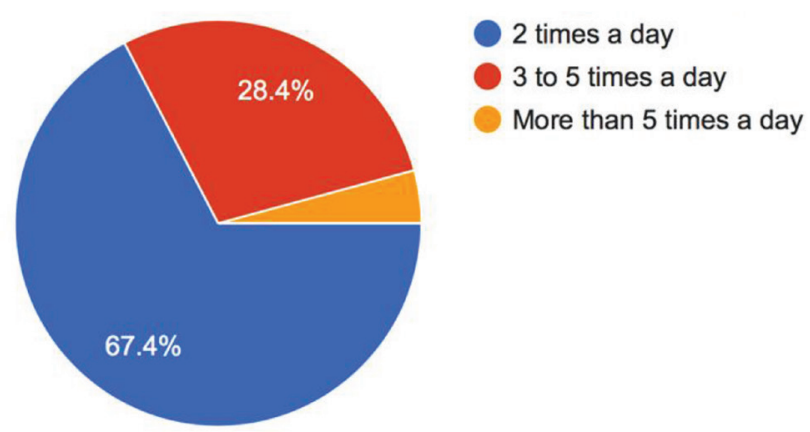

Fig. 3 Frequency of tea or coffee consumption.
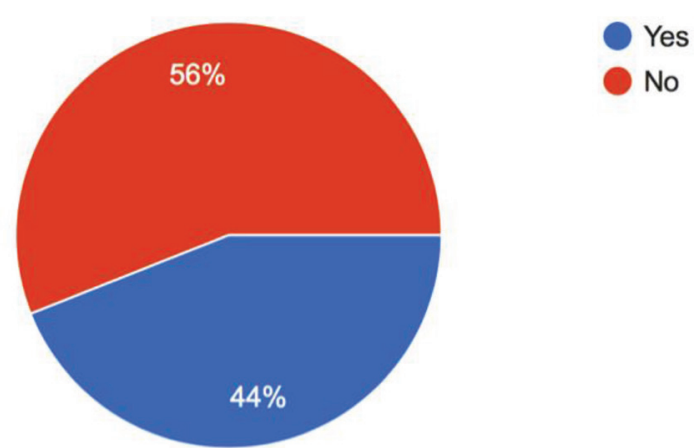

Fig. 4 Consumption of aerated beverages.

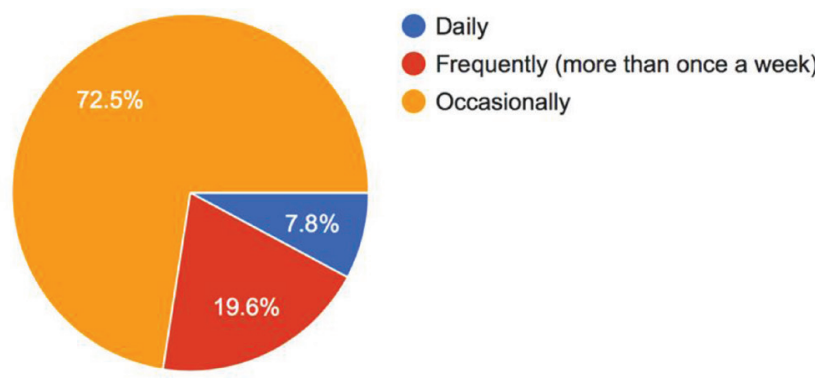

Fig. 5 Frequency of aerated beverage consumption. percent of the patients reported alcohol consumption habits. Tobacco chewing habit was reported by $7 \%$ of the patients. Thirteen percent of the patients reported cigarette smoking habit. Thirty-three percent of the patients reported no alcohol or tobacco consumption habits ( - Fig. 6). Of the patients reporting alcohol and tobacco consumption, $51.4 \%$ of the patients reported to be consuming them occasionally whereas $38.6 \%$ of the patients reported consuming them frequently and $10 \%$ of the patients reported consuming them daily ( - Fig. 7). Almost $96 \%$ of the patients cleaned their dentures using some or the other method ( - Fig. 8). Almost $55 \%$ of the patients claimed to be cleaning their dentures once daily and approximately $35 \%$ of the patients reported to be cleaning their dentures two times a day, whereas around $10 \%$ of the patients cleaned their dentures only every alternate day ( - Fig. 9). About $16 \%$ of the patients used detergents with water to clean their dentures, approximately $21 \%$ of the patients used toothpaste to clean their dentures, and almost $38 \%$ of the patients used only water to rinse and clean their dentures, whereas only approximately

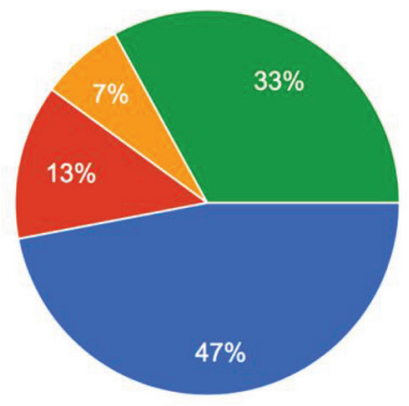

Alcohol consumption

Cigarette smoking

Tobacco chewing

None of the above

Fig. 6 Alcohol and tobacco consumption.

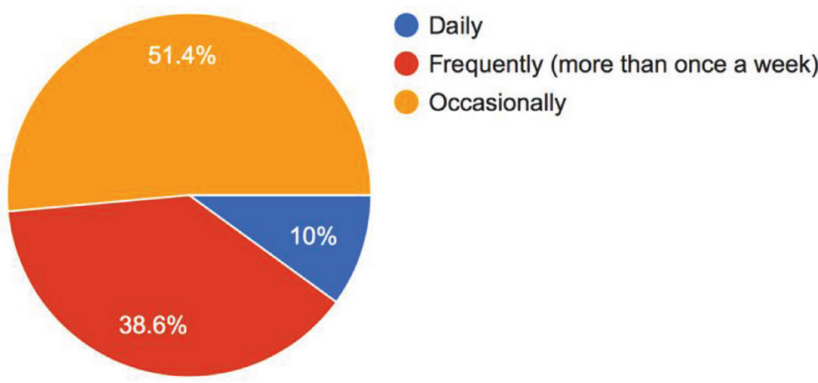

Fig. 7 Frequency of alcohol and tobacco consumption.

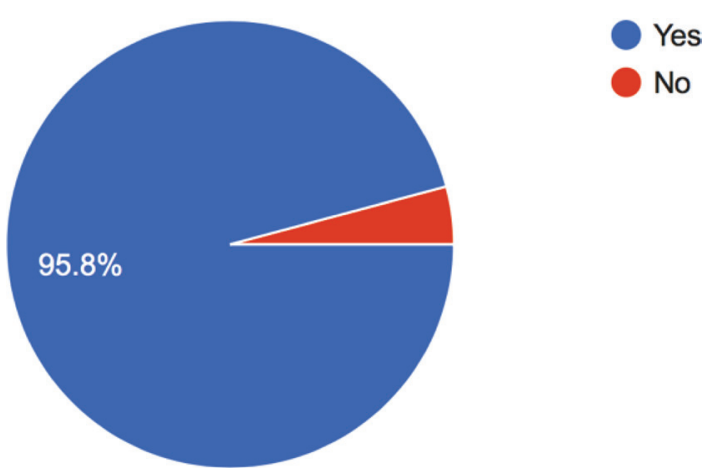

Fig. 8 Denture cleaning. 
$25 \%$ of the patients used appropriate denture cleaning solu-

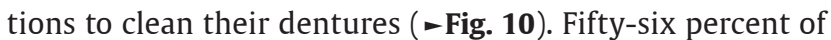
the patients claimed to be aware of some or the other denture cleaning techniques, whereas $44 \%$ were not aware of any denture cleaning techniques (-Fig. 11). About $42 \%$ of the patients who cleaned their dentures soaked them overnight in salt water, and approximately 55\% of the patients used appropriate denture cleaning solutions, whereas only approximately $3 \%$ of the patients sought professional denture cleaning by a dental technician or a dentist ( - Fig. 12). About $77 \%$ of the patients complained of halitosis (bad breath), $15 \%$ of the patients complained of oral ulcers, and approximately $8 \%$ of the patients suffered from oral
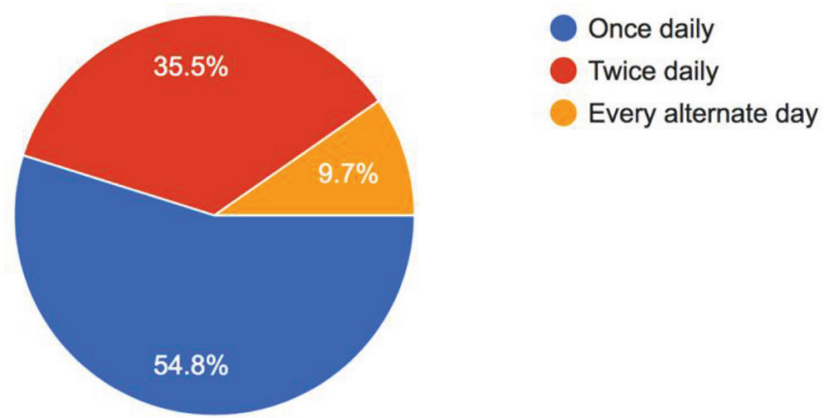

Fig. 9 Frequency of denture cleaning.

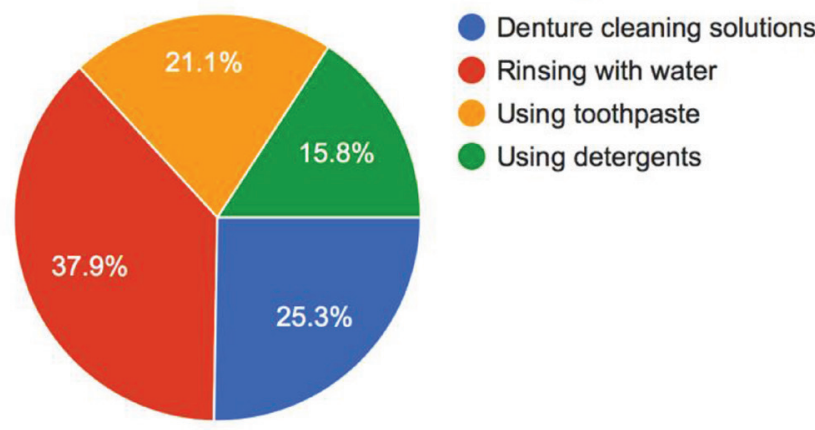

Fig. 10 Denture cleaning methods used.

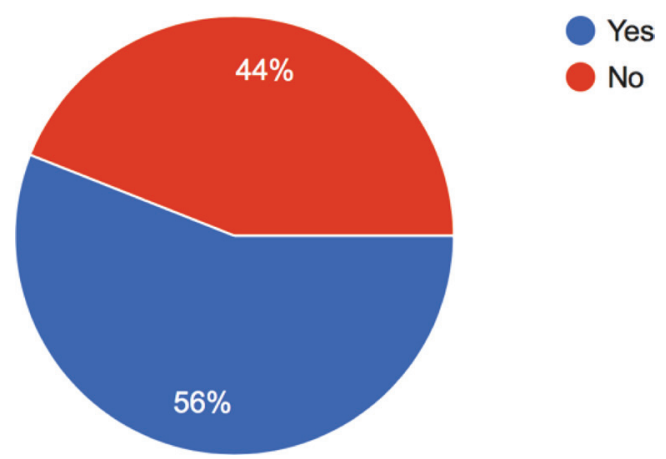

Fig. 11 Awareness of denture cleaning techniques.

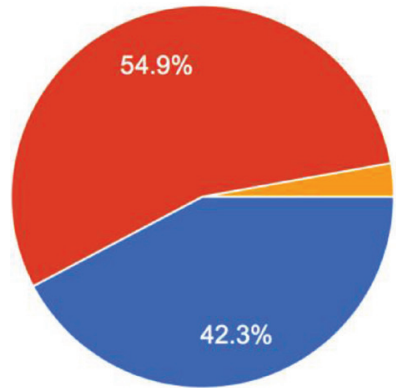

Soaking in salt water overnight

Using denture cleaning solutions

Professional cleaning by a dental technician

Fig. 12 Knowledge of specific denture cleaning techniques.

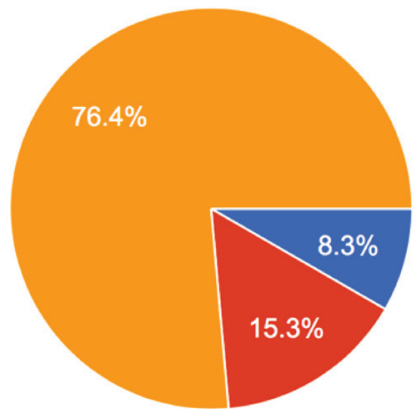

Oral infections

Oral ulcers

Halitosis (bad breath)

Fig. 13 Ailments related to oral hygiene maintenance.

infections (-Fig. 13). About $31 \%$ of the patients claimed to be aware and $69 \%$ of the patients were completely unaware of any association between denture hygiene maintenance and oral infections or ulcers or bad breath ( - Fig. 14). About $63 \%$ of the patients were satisfied with their quality of denture maintenance, whereas $37 \%$ of the patients were unsatisfied with their denture maintenance ( - Fig. 15).

\section{Discussion}

Knowledge and awareness go hand in hand, as one cannot be without the other. The treatment rendered to patients is almost always incomplete without proper education and motivation that our patients require to ensure a problem-free, functional and aesthetic prosthesis with ensured longevity. ${ }^{5}$

While most of the patients in the current study practiced cleaning their dentures regularly, a majority of this group cleaned them simply by rinsing the dentures with water whereas only small group of these patients used commercially available denture cleaning solutions. This is a direct reflection of the lack of awareness regarding prosthetic and oral hygiene maintenance among the patients. That and the fact that almost half of the patients claimed to be unaware of any denture cleaning methods, highlights the need to educate and motivate our patients to the best of our abilities to ensure a satisfactory treatment in the long run. Almost half of the patients cleaned the dentures once daily, where this 


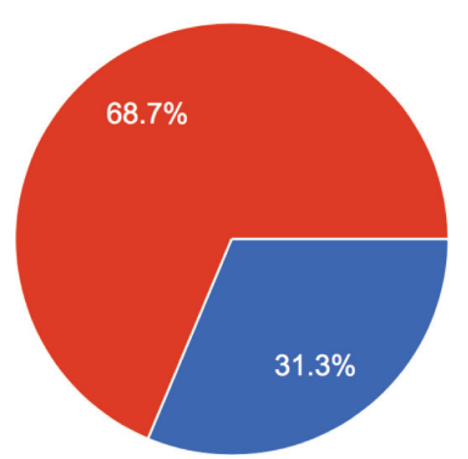

Fig. 14 Awareness of link between denture hygiene and ailments.

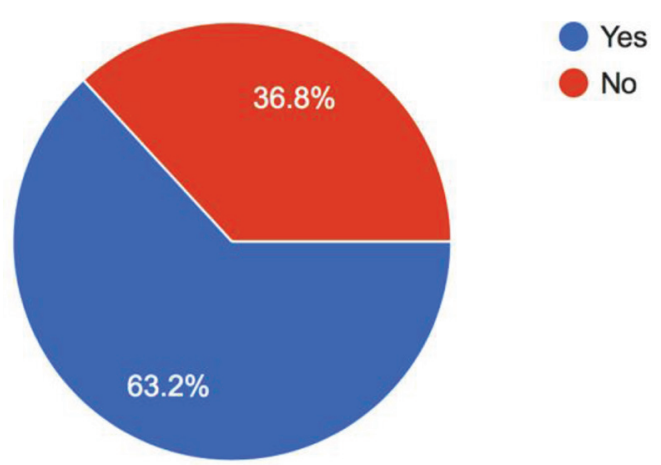

Fig. 15 Denture maintenance satisfaction.

result can be compared with that of Nevalainen et al who obtained similar results, some of the patients cleaned the dentures twice a day and a few even cleaned once in every 2 days. This shows that many of them were prone to denture staining and a few were also wearing stained dentures probably due to lack of awareness. ${ }^{6-11}$

A disclosing agent is recommended to be used to make the plaque visible on the denture surface. Excessive wear should be avoided and a paste with low abrasive content (polymethyl methacrylate or sodium bicarbonate) and a soft denture brush or toothbrush should be used. Brushing should be done meticulously, because denture plaque cannot be easily removed. Efficient chemical denture cleansers can serve as better alternatives to mechanical cleansing, especially among geriatric or handicapped denture wearers. Daily, overnight immersion of dentures in an alkaline peroxide solution provides for a safe and relatively effective method of cleaning dentures. These products do not have any effect when only immersed for 15 to 30 minutes, as recommended by the manufacturers. Hypochlorite cleansers are effective with overnight immersion, but because of bleaching, they should be used only intermittently, for example, once a week. ${ }^{12,13}$

For better denture cleaning, all clinical and laboratory procedures used during prosthodontic treatment and denture fabrication should aim at providing dentures with a smooth and homogenous surface. However, the tissue surface of dentures usually shows micropits and microporosities which harbor microorganisms which are difficult to remove by mechanical or chemical cleaning. It has also been shown that polishing of the denture's tissue surface is favorable to improved denture hygiene and healing of denture stomatitis and also does not affect retention of the dentures, indicating that polishing of the denture's tissue surface can be considered as a routine step in prosthodontic treatment.

Most of the patients reported having either bad breath (halitosis) or oral ulcers or oral infections currently or in the past while using their dentures. Despite having suffered such adversities, most of the patients did not know that stained and unhygienic dentures have any link with these ailments. This is probably due to not just the lack of awareness of denture hygiene but also ignorance of the patients with respect to their own ailments.

Denture maintenance goes a long way in ensuring long-lasting successful dentures for our patients. A large number of patients were unsatisfied with their denture hygiene maintenance. This is mostly due to lack of knowledge and awareness of oral and denture hygiene and the ignorance toward one's own health.

\section{Conclusion}

Within the limitations of this study, it was concluded that knowledge and awareness on oral and denture hygiene still remains questionable among a majority of our denture wearers. We as practitioners should give our best efforts to educate our patients regarding oral and denture hygiene practices, various food and beverage consumption, adverse oral habits, and their consequences on dentures. We should motivate our patients to take greater care of their prosthesis and visit a dentist for professional cleaning of their dentures regularly. Conforming to such healthy practices will go a long way in providing utmost satisfaction to our patients as denture wearers and us as practitioners.

\section{Conflict of Interest}

None.

\section{References}

1 Barber S, Jerreat M, Jagger DC, Harrison A. Denture care of patients in a general hospital. J Disability Oral Health 2002;3:68-71

2 Peracini A, Andrade IM, Paranhos HdeF, Silva CH, de Souza RF. Behaviors and hygiene habits of complete denture wearers. Braz Dent J 2010;21(3):247-252

3 Dikbas I, Koksal T, Calikkocaoglu S. Investigation of the cleanliness of dentures in a university hospital. Int J Prosthodont 2006;19(3):294-298

4 Roshene R, Robin P, Raj JD. A survey of denture hygiene in older patients. J Pharmaceut Sci Res 2015;7(10):897-900

5 Murtomaa $\mathrm{H}$, Könönen $\mathrm{M}$, Laine $\mathrm{P}$. Age and maintenance of removable dentures in Finland. J Oral Rehabil 1992;19(2):123-128 
6 Suresan V, Mantri S, Deogade S, et al. Denture hygiene knowledge, attitudes, and practices toward patient education in denture care among dental practitioners of Jabalpur city, Madhya Pradesh, India. J Indian Prosthodont Soc 2016;16(1):30-35

7 Kulak-Ozkan Y, Kazazoglu E, Arikan A. Oral hygiene habits, denture cleanliness, presence of yeasts and stomatitis in elderly people. J Oral Rehabil 2002;29(3):300-304

8 Paranhos HdeF, da Silva CH, Venezian GC, Macedo LD, de Souza RF. Distribution of biofilm on internal and external surfaces of upper complete dentures: the effect of hygiene instruction. Gerodontology 2007;24(3):162-168

9 de Castellucci Barbosa L, Ferreira MR, de Carvalho Calabrich CF, Viana AC, de Lemos MC, Lauria RA. Edentulous patients' knowledge of dental hygiene and care of prostheses. Gerodontology 2008;25(2):99-106

10 Shreya S, Sangeetha Kirubagaran S. A questionnaire based survey among the patients wearing complete dentures on denture staining. Int J Sci Res (Ahmedabad) 2016;5(6):591-596

11 Nevalainen MJ, Närhi TO, Ainamo A. Oral mucosal lesions and oral hygiene habits in the home-living elderly. J Oral Rehabil 1997;24(5):332-337

12 Jeganathan S, Payne JA, Thean HP. Denture stomatitis in an elderly edentulous Asian population. J Oral Rehabil 1997;24(6):468-472

13 Budtz-Jørgensen E. Materials and methods for cleaning dentures. J Prosthet Dent 1979;42(6):619-623 